Winter 2018

\title{
Advanced Magnetic Resonance Imaging in Glioblastoma: A Review
}

\author{
Gaurav Shukla \\ Thomas Jefferson University \\ G. S. Alexander \\ Thomas Jefferson University \\ Spyridon Bakas \\ University of Pennsylvania \\ Rahul Nikam \\ Thomas Jefferson University \\ Kiran Talekar \\ Thomas Jefferson University
}

Follow this and additional works at: https://jdc.jefferson.edu/jhnj

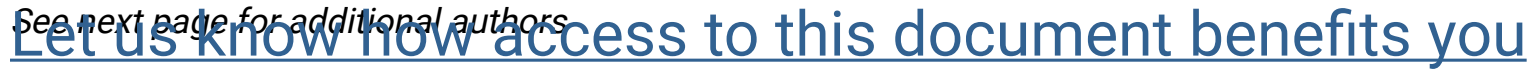

\section{Recommended Citation}

Shukla, Gaurav; Alexander, G. S.; Bakas, Spyridon; Nikam, Rahul; Talekar, Kiran; Palmer, Joshua; and Shi, Wenyin (2018) "Advanced Magnetic Resonance Imaging in Glioblastoma: A Review," JHN Journal: Vol. 13 : Iss. 1 , Article 5.

DOI: https://doi.org/10.29046/JHNJ.013.1.005

Available at: https://jdc.jefferson.edu/jhnj/vol13/iss1/5

This Article is brought to you for free and open access by the Jefferson Digital Commons. The Jefferson Digital Commons is a service of Thomas Jefferson University's Center for Teaching and Learning (CTL). The Commons is a showcase for Jefferson books and journals, peer-reviewed scholarly publications, unique historical collections from the University archives, and teaching tools. The Jefferson Digital Commons allows researchers and interested readers anywhere in the world to learn about and keep up to date with Jefferson scholarship. This article has been accepted for inclusion in JHN Journal by an authorized administrator of the Jefferson Digital Commons. For more information, please contact: JeffersonDigitalCommons@jefferson.edu. 


\section{Advanced Magnetic Resonance Imaging in Glioblastoma: A Review}

\section{Authors}

Gaurav Shukla, G. S. Alexander, Spyridon Bakas, Rahul Nikam, Kiran Talekar, Joshua Palmer, and Wenyin Shi 


\section{Advanced Magnetic Resonance Imaging in Glioblastoma: A Review}

\author{
Gaurav Shukla, MD1,2; G.S. Alexander, BS1; Spyridon Bakas, $\mathrm{PhD}^{2,3}$; Rahul Nikam, \\ MD4; Kiran Talekar, MD4; Joshua Palmer, MD5; Wenyin Shi, MD1 \\ 'Department of Radiation Oncology, Thomas Jefferson University, Sidney Kimmel Cancer \\ Center at Jefferson, Philadelphia, PA \\ ${ }^{2}$ Center for Biomedical Image Computing and Analytics, University of Pennsylvania, \\ Philadelphia, PA \\ ${ }^{3}$ Department of Radiology, University of Pennsylvania, Philadelphia, PA \\ ${ }^{4}$ Department of Radiology, Thomas Jefferson University, Philadelphia, PA \\ Department of Radiation Oncology, The Ohio State University Wexner Medical Center, \\ Columbus, $\mathrm{OH}$
}

\section{INTRODUCTION}

In 2017, it is estimated that 26,070 patients will be diagnosed with a malignant primary brain tumor in the United States, with more than half having the diagnosis of glioblastoma (GBM). ${ }^{1}$ Magnetic resonance imaging (MRI) is a widely utilized examination in the diagnosis and post-treatment management of patients with glioblastoma; standard modalities available from any clinical MRI scanner, including T1, T2, T2-FLAIR, and T1-contrast-enhanced (T1CE) sequences, provide critical clinical information. In the last decade, advanced imaging modalities are increasingly utilized to further characterize glioblastomas. These include multi-parametric MRI sequences, such as dynamic contrast enhancement (DCE), dynamic susceptibility contrast (DSC), diffusion tensor imaging (DTI), functional imaging, and spectroscopy (MRS), to further characterize glioblastomas, and significant efforts are ongoing to implement these advanced imaging modalities into improved clinical workflows and personalized therapy approaches. A contemporary review of standard and advanced MR imaging in clinical neuro-oncologic practice is presented.

\section{Initial diagnosis and surgical management}

Most patients with glioblastoma undergo computed tomography of the brain upon initial presentation. Once a mass is identified and hemorrhage is excluded, a contrastenhanced MRI is typically ordered, with standard T1, T2, FLAIR, and contrast-enhanced T1 (T1CE) sequences. ${ }^{2,3}$ Many institutions will also capture gradient echo and diffusion sequences. Maximal safe debulking surgery is recommended as the initial standard of care. Neurosurgeons will often utilize high-resolution MRI $(0.5-1.2 \mathrm{~mm}$ slice thickness) for surgical planning and intraoperative guidance, as well as to make the determination of how aggressively to resect based on risk of toxicity to nearby eloquent regions. ${ }^{4}$ Standard imaging also can identify other important characteristics of the mass in situ, including the amount of necrosis, compression of the surrounding normal tissue, and midline deviation.

A recent meta-analysis of over 40,000 glioblastoma patients demonstrated that grosstotal resection was associated with improved survival as compared to subtotal resection. ${ }^{5}$ Historically, the determination of gross-total resection was made in the operating room by the neurosurgeon. However, in the modern era, the practice of obtaining a postoperative contrast-enhanced MRI within 24-48 hours of surgery has become routine after publication of a study showing that radiological determination of the extent of resection via MRI had prognostic significance. ${ }^{6}$ Several series have attempted to quantify a threshold value for the extent of resection as a guide for neurosurgeons, utilizing the amount or enhancing tumor present in the preoperative and post-operative T1CE images. These series report thresholds ranging from $70 \%$ to $100 \%^{7-9}$, with the caveats that they were obtained retrospectively. To date, no formal threshold is recommended other than "maximal safe resection" as mentioned previously.

Standard preoperative images can be analyzed for macroscopic shape and location features that are associated with improved survival, ${ }^{10-13}$ providing potential biomarkers that may be utilized in stratifying patients in clinical trials.

Advanced MR imaging sequences have utility in the preoperative domain as well. Functional imaging (fMRI) has been particularly useful in preoperative surgical planning in cases where tumors or their resection may disrupt eloquent areas. Many patients who were once felt to be unresectable due to uncertain risk of neurologic compromise are now candidates for more aggressive resection after functional mapping. ${ }^{14}$ Diffusion tensor imaging (DTI) generates rich white matter tractography images which may guide neurosurgical planning $^{15}$ and can help distinguish between post-operative vascular damage and residual enhancing tumor. ${ }^{16}$ Dynamic contrast-enhanced (DCE) sequences in the preoperative setting measure pharmacokinetic parameters of contrast uptake, which may be associated with early disease progression and survival. ${ }^{17}$ Dynamic susceptibility contrast (DSC) MR imaging may be helpful in preoperative diagnosis ${ }^{18}$ of malignant lesions. Imaging features extracted from standard and advanced preoperative MR sequences can predict survival, molecular subtype, and mutational status in glioblastoma, 19,20 potentially enhancing the set of imaging biomarkers available to clinicians.

\section{Post-operative imaging and radiation planning}

After maximal safe resection, which is evaluated on immediate post-operative MRI, the standard of care for patients with glioblastoma is chemoradiation with concurrent temozolomide, after the results of a large randomized Phase III trial. ${ }^{21}$ Typically, chemoradiation begins 3-6 weeks after surgery to allow 


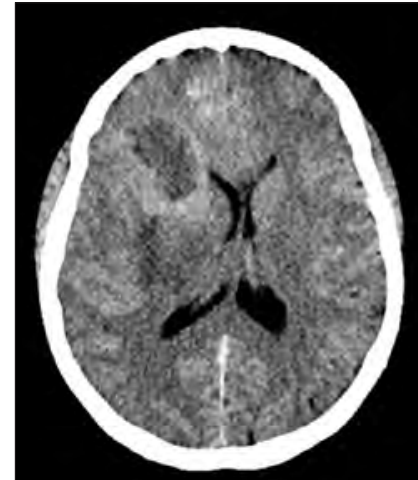

Figure 1

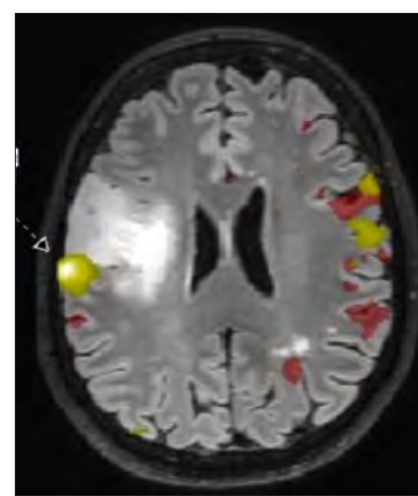

Figure 5

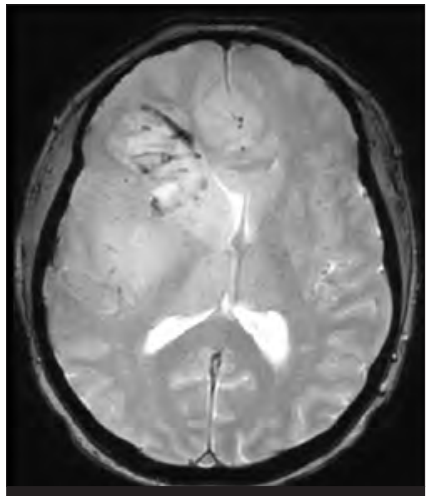

Figure 2

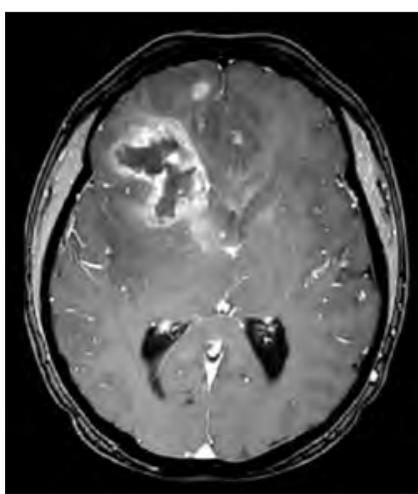

Figure 6

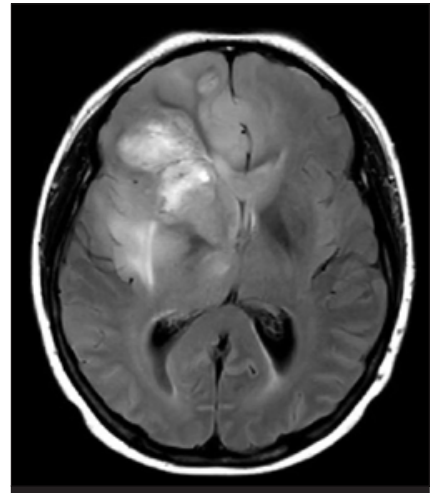

Figure 3

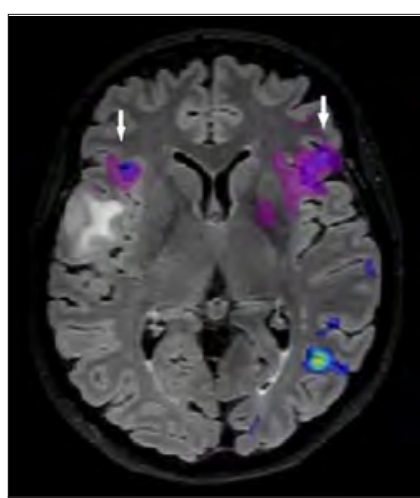

Figure 7
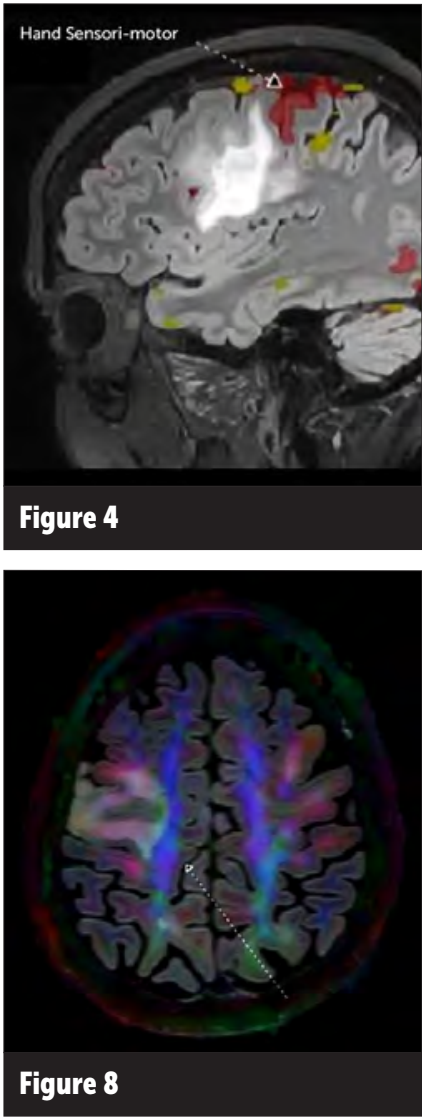

Figure 1. Axial CT image at the level of basal ganglia demonstrates a large heterogeneous mass in the right frontal lobe with mass effect on the right lateral ventricle and leftward shift of midline. $\mathrm{Ct}$, computed tomography.

Figure 2. Axial FLAIR weighted image at the level of basal ganglia demonstrates heterogeneous mass centered in the right frontal lobe and basal ganglia with surrounding infiltrating signal abnormality 'FLAIR envelope' which extends medially across the corpus callosum posteriorly in the insular region. The 'FLAIR envelope' is typically a manifestation of combination of tumor infiltration and edema. There is associated mass effect on the right ventricle and leftward midline shift. FLAIR, fluid-attenuated inversion recovery.

Figure 3. Axial gradient echo (GRE) image depicts multiple foci of hypointense signal 'susceptibility artifacts' within the right frontal mass compatible with intra-tumoral blood products.

Figure 4. Post gadolinium based contrast administration $\mathrm{T} 1$ weighted axial image (T1CE). There is heterogeneous irregular peripheral enhancement associated with the right frontal lobe mass with central non-enhancing area, consistent with necrosis. Of note are additional patchy areas of enhancement in the right anterior frontal lobe and right basal ganglia region. These additional areas of enhancement lie within the previously described region of 'FLAIR envelope'. FLAIR, fluid-attenuated inversion recovery; T1CE, T1 contrast-enhanced.

Figure 5. BOLD fMRI for localization of hand sensorimotor cortex in a patient with right frontal glial neoplasm. BOLD fMRI data is superimposed on sagittal FLAIR weighted image for anatomic localization. In the right hemisphere, the hand sensorimotor cortex (arrow) is located along the posterosuperior aspect of the frontal mass and is separated by less than one gyrus distance. fMRI, functional magnetic resonance imaging; FLAIR, fluidattenuated inversion recovery.

Figure 6. BOLD fMRI for localization of tongue sensorimotor cortex. BOLD fMRI data is superimposed on axial FLAIR weighted image for anatomic localization. In the right hemisphere, the area of activation (arrow), tongue sensorimotor cortex is in immediate proximity of the posterior margin of the right frontal mass. FLAIR envelope seems to extend into this region of activation. fMRI, functional magnetic resonance imaging; FLAIR, fluid-attenuated inversion recovery.

Figure 7. BOLD fMRI for localization of Broca's area in a patient with right frontal glial neoplasm. There is bilateral Broca's area activation on sentence completion and verb generation tasks (arrows), with the right hemispheric area of activation located at the anteroinferior aspect of tumor within one gyrus distance. $\mathrm{fMRI}$, functional magnetic resonance imaging.

Figure 8. Color fractional anisotropy map superimposed on axial FLAIR weighted image. There is loss of fractional anisotropy in the expected region of right corticospinal tract (arrow, blue colored fibers). This tract is located at the posteromedial margin of the FLAIR envelope. Loss of fractional anisotropy may be related edema, infiltration by tumor or displacement. FLAIR, fluid-attenuated inversion recovery. 


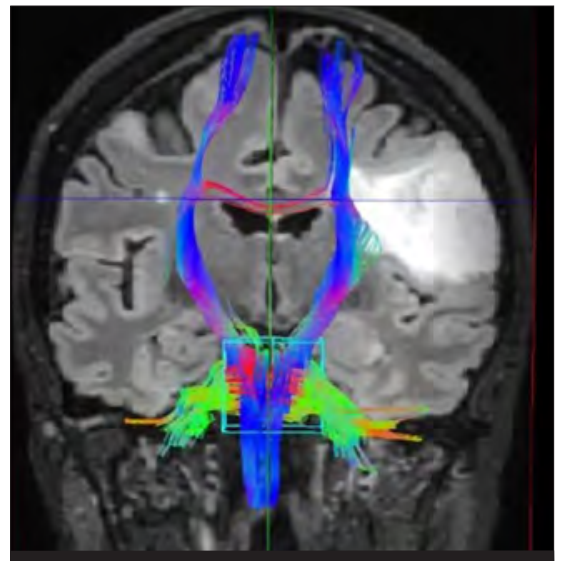

Figure 9.

Tractography image demonstrates the intimate relationship of right frontal mass with the corticospinal tract (blue colored fibers).

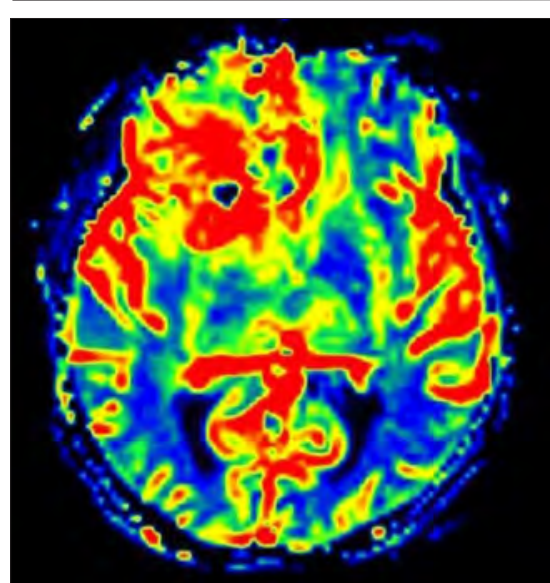

Figure 10.

Dynamic susceptibility contrast (DSC) perfusion weighted image. There is increase in rCBV (relative cerebral blood volume) in the region of right frontal mass (Figures 1-4), a finding favoring high grade neoplasm.

for adequate post-operative recovery. Radiotherapy planning includes registration (aka "fusion") of the post-operative MRI (T1CE and FLAIR sequences) with the planning simulation $\mathrm{CT}$, which allows for delineation of the FLAIR abnormality and residual enhancement in treatment planning. Guidelines for these delineations exist, but substantial variation is observed among practitioners from different cooperative groups (e.g., RTOG ${ }^{22}$ vs. EORTC ${ }^{23}$ ), and even among practitioners from one country, ${ }^{24}$ but all utilize post-operative

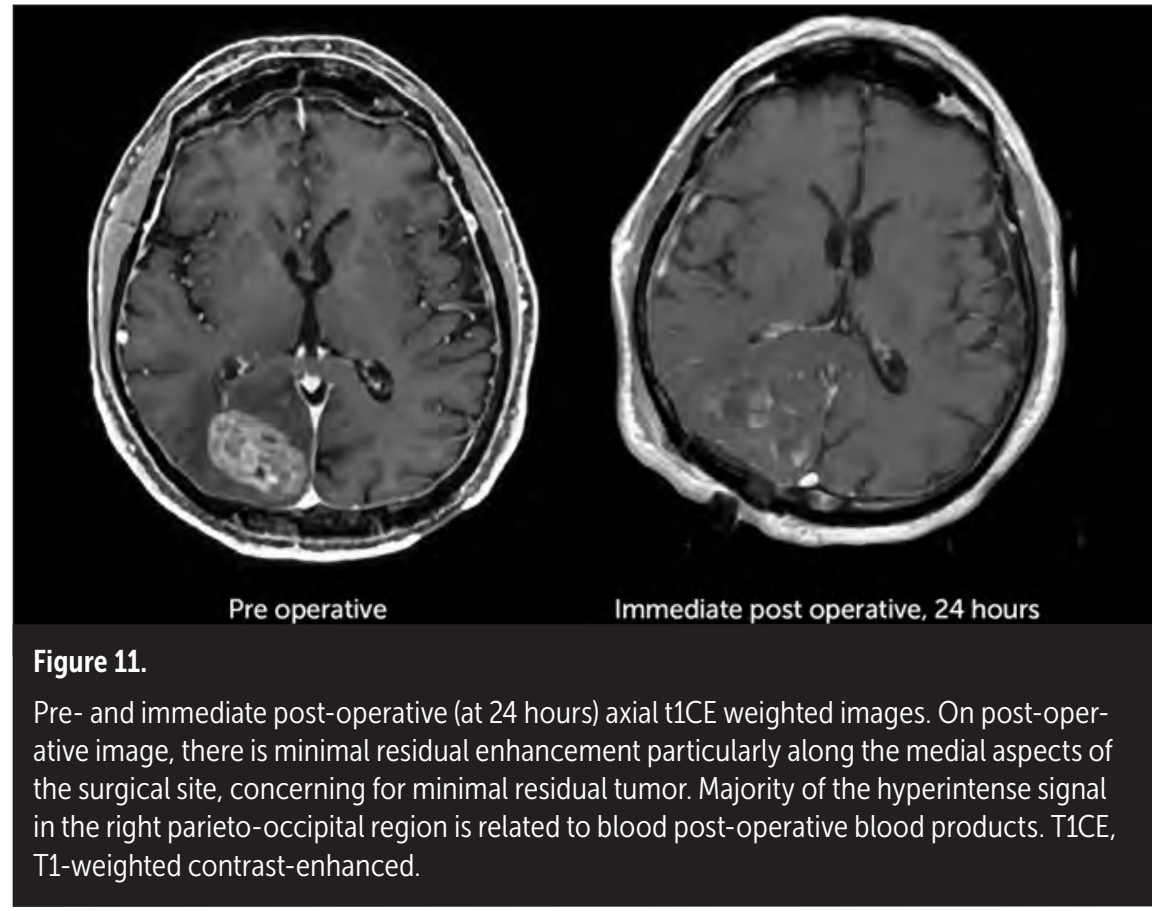

MR imaging to define the at risk target volumes and organs at risk.

It is common to identify shifting of brain parenchyma on planning CT in the weeks after craniotomy as the normal brain tissue expands to fill the space taken out by the tumor. One study demonstrated a $4 \mathrm{~mm}$ shift in the position of the treatment isocenter between CT and MRI-based target delineation, ${ }^{25}$ even with only a few days between studies. The magnitude of the shift can be several centimeters, resulting in inaccurate registration between post-operative MRI and simulation CT. Many institutions have begun the practice of obtaining repeat MRI at the time of simulation to better characterize the soft tissues for target delineation.

Advanced imaging at this time point may play a role in radiation planning. A Polish study demonstrated the discordance between gross tumor volume (GTVs) delineated from MRI as compared to 18F-fluoroethylthyrosine-PET (FET-PET), a functional imaging modality; FET-PET was better associated with the site of eventual failure, suggesting that traditional target volumes may not be adequate. ${ }^{26}$ ADC maps generated from diffusion imaging can identify areas of restricted diffusion that may predict for areas of eventual recurrence with high concordance; 27,28 along with fractional anisotropy measurements from diffusion images, ADC values may be associated with poor response to treatment and worse survival among high grade glioma patients. ${ }^{29}$ Diffusion and perfusion parameters, when combined with standard MR sequences, may allow radiation oncologists to better characterize the highest-risk regions to include in high-dose target volumes, utilizing macroscopically visible features ${ }^{30}$ as well as radiomic features. ${ }^{31}$ Voxel-based MR spectroscopy (MRS) and whole-brain spectroscopic MRI (sMRI) may identify regions of tumor infiltration and areas at high risk of recurrence; ${ }^{32}$ regions with metabolic abnormalities on sMRI are correlated with intraoperative tissue samples showing increased immunohistochemical staining for neoplastic cells. ${ }^{33}$

\section{Response Assessment}

As demonstrated at any multidisciplinary tumor board, imaging is of utmost importance in the interpretation of the response to treatment in glioblastoma. The first widelyadopted set of guidelines for standardizing the assessment of treatment response that utilized MR imaging was the Macdonald criteria, ${ }^{34}$ which used clinical parameters in conjunction with imaging measurements to classify responses into four broad categories (complete response, partial response, stable disease, and progressive disease). 


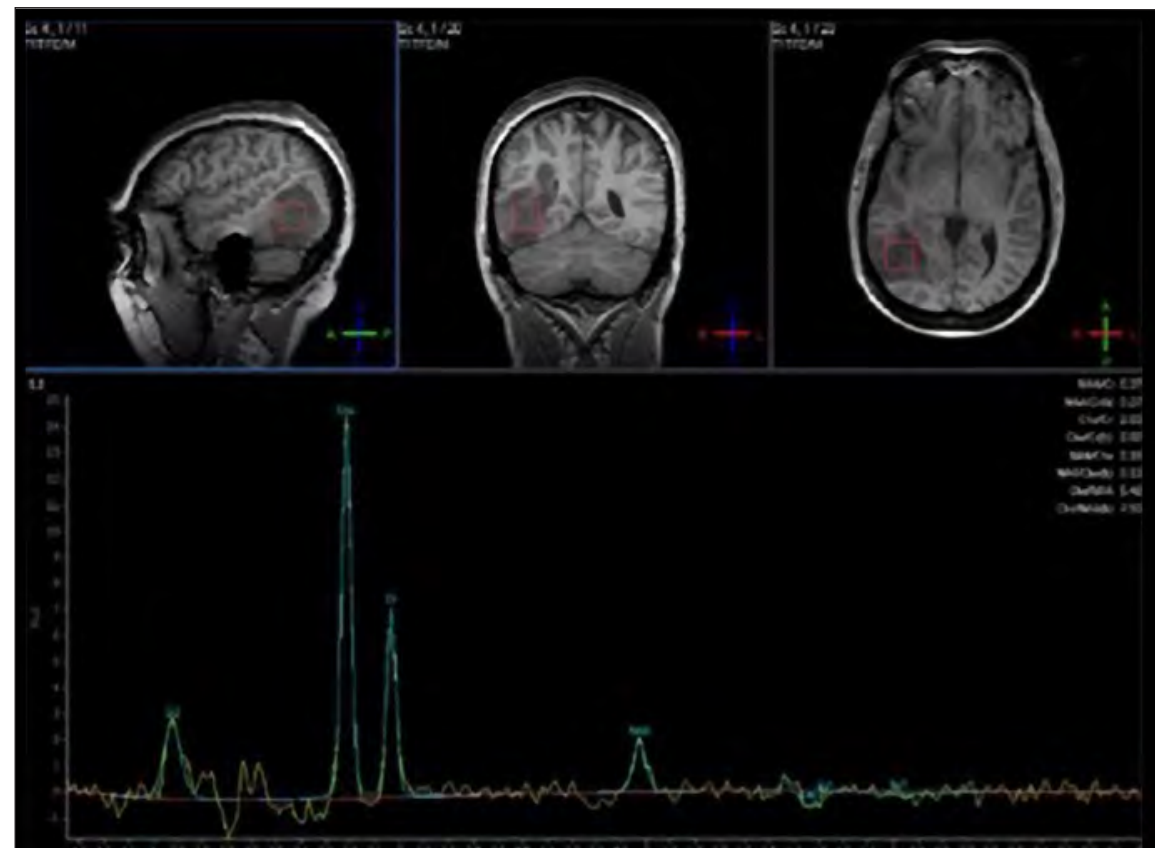

\section{Figure 12.}

Single voxel MR spectroscopy at long TE (288 ms) acquired through the right temporoparietal region mass with imaging appearance compatible with glial neoplasm. There is markedly elevated choline (resonates at $3.2 \mathrm{ppm}$ ) with markedly decreased NAA (resonates at $2 \mathrm{ppm}$ ), a finding consistent with high grade glial neoplasm. MR, magnetic resonance.

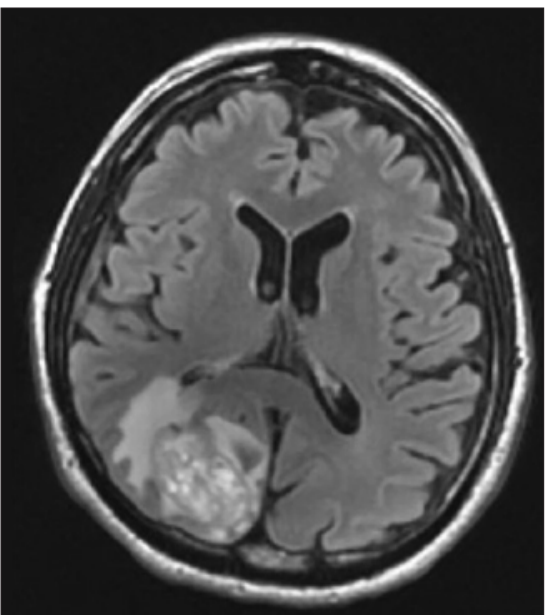

Figure 13.

Axial FLAIR and post contrast T1 weighted images demonstrate a large heterogeneously enhancing mass in the right parieto-occipital region with surrounding FLAIR hyperintense signal, compatible with high grade glial neoplasm. FLAIL, fluid-attenuated inversion recovery.

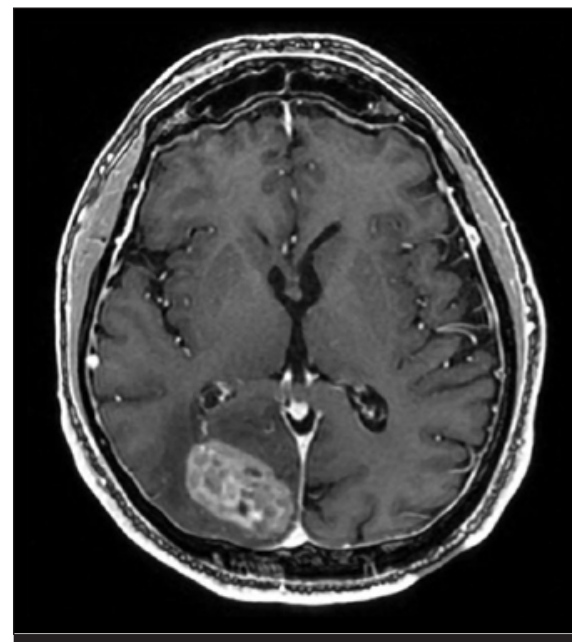

Figure 14.

Immediate post-operative

(at 24 hours) axial post contrast T1 weighted image. There is minimal residual peripheral enhancement particularly along the medial aspects of the surgical site concerning for small amount of residual tumor.

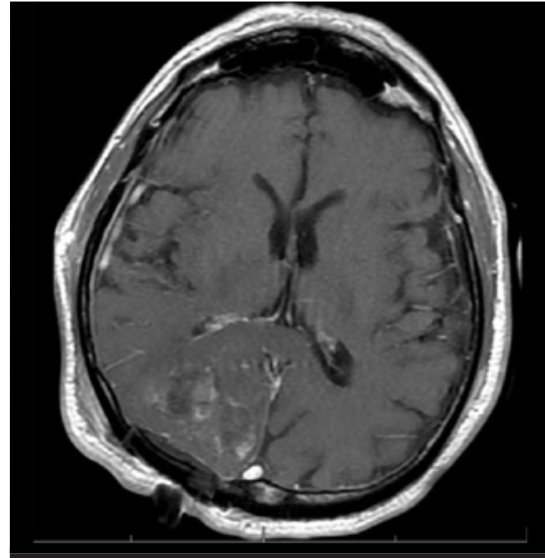

Figure 15.

Immediate post-operative (at 24 hours) axial post contrast $\mathrm{T1}$ weighted image. There is minimal residual peripheral enhancement particularly along the medial aspects of the surgical site concerning for small amount of residual tumor.

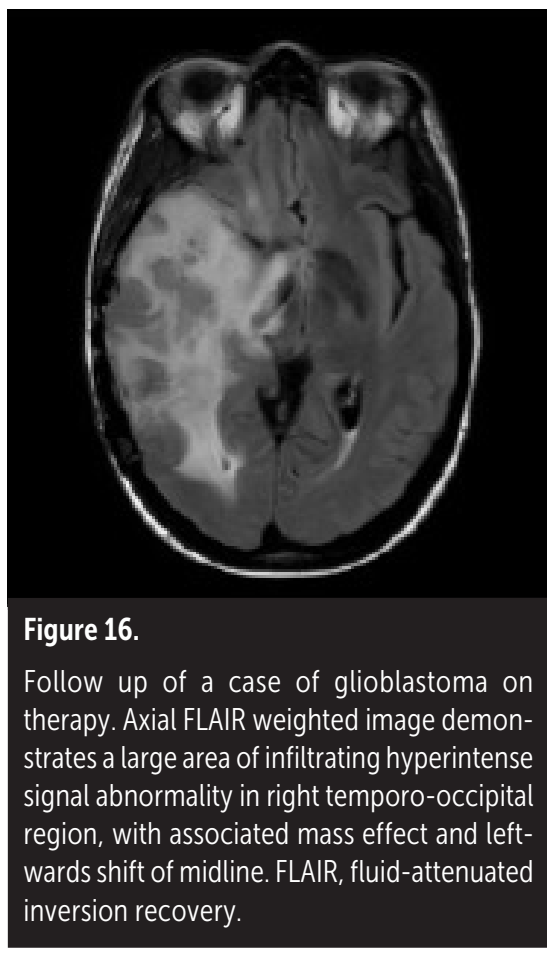

Challenges and limitations of the Macdonald criteria became apparent as imaging modalities revealed more details about gliomas and their response to treatment. The importance of noncontrast-enhancing regions of abnormality has become better understood; for example, changes in the volume of 


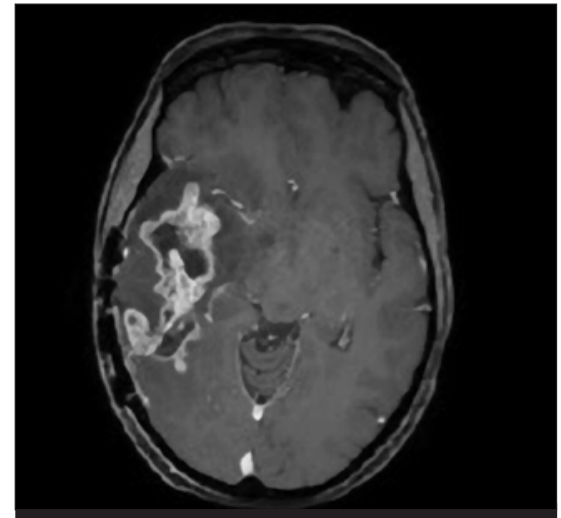

Figure 17.

Axial T1CE image depicts an area of heterogeneous enhancement in right temporal lobe within the region of FLAIR signal abnormality. FLAIR, fluid-attenuated inversion recovery. T1CE, T1-weighted contrast-enhanced

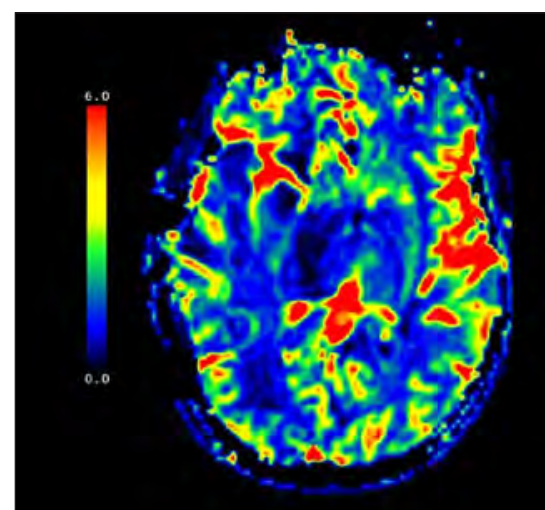

Figure 19.

On dynamic susceptibility contrast (DSC) perfusion weighted imaging, the area of signal abnormality predominantly demonstrates low relative cerebral blood volumes. The overall findings were consistent with pseudoprogression.

hyperintensity on post-treatment FLAIR imaging, relative to baseline, are correlated with improved survival. ${ }^{35}$ Furthermore, some glioblastomas demonstrate imaging changes consistent with progression under the Macdonald criteria, but upon repeat surgical intervention, viable tumor cannot be identified in the resection specimen, suggesting that the adjuvant treatment may actually be having a positive effect that eludes detection on conventional imaging. This finding, termed "pseudoprogression,"

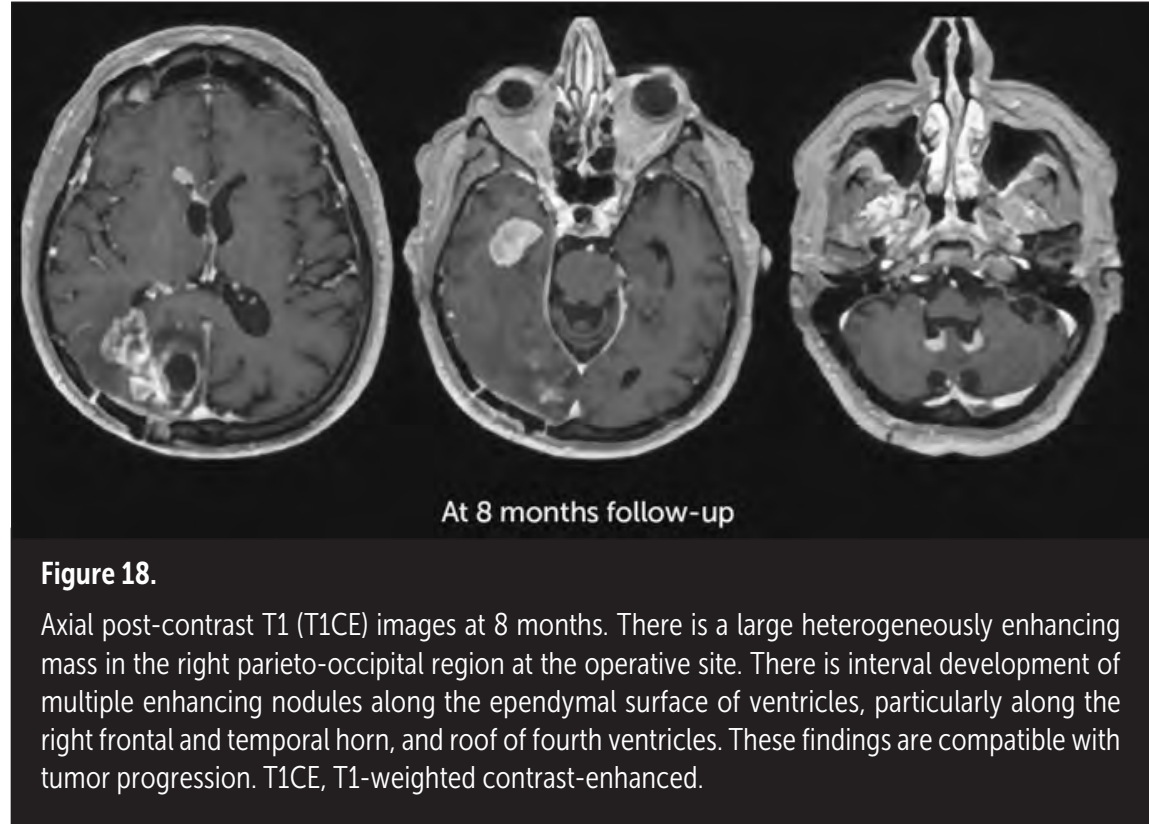

is most commonly observed in patients whose tumors harbored a methylated MGMT promoter region, ${ }^{36}$ and makes accurate assessment of response difficult, especially in the setting of clinical trials attempting to answer the question of efficacy of novel treatment regimens. Some medications, including anti-angiogenic drugs and immunologic agents, elicit unique radiographic changes which may mask accurate response assessment as well.

These limitations, among others, led to the development of a new set of guidelines developed by the Response Assessment in Neuro-Oncology (RANO) working group, ${ }^{37}$ which incorporates more information from MR imaging, including FLAIR sequence changes, into the objective assessment. The RANO criteria have been incorporated into clinical trials and daily clinical practice, allowing better applesto-apples comparisons. ${ }^{38}$

Clinical trials in the last decade demonstrated the benefit of bevacizumab, an anti-angiogenic monoclonal antibody, in recurrent glioblastoma. ${ }^{39}$ The radiographic appearance of malignant gliomas changes dramatically after treatment with bevacizumab as a result of changes in vessel permeability and contrast dynamics. ${ }^{40}$ Initial studies showed the difficulty in distinguishing these radiographic changes from true tumor effect; the temporal dynamics were also unclear. ${ }^{41}$ These issues led to the development of the immunotherapy response assessment in neuro-oncology (iRANO) criteria, ${ }^{42}$ which attempted to provide standardized guidelines for the determination of tumor progression in the setting of immunerelated therapy.

MRI imaging features have the potential to predict treatment response to specific modalities of treatment. Relative cerebral blood volume and dynamics parameters ( $\mathrm{K} \neg$ trans and $V e)$, measured by perfusion-weighted MR imaging and other features may predict treatment response to standard chemoradiation and VEGF inhibitors, ${ }^{43-45}$ prior to initiation of therapy. Radiomic features derived from these images have been shown to have predictive value as well. ${ }^{46}$

\section{CONCLUSIONS}

The volume of medical imaging data continues to grow at an exponential rate. As MR imaging becomes more cost-effective and the adoption of advanced MR modalities becomes more widespread, it will become more critical than ever to incorporate advanced imaging and the power of large datasets into the management of glioblastoma. We anticipate that these changes will include not only the utilization of new MR sequences but also novel image analysis techniques, including radiomic analysis, to better drive treatment decisionmaking, with the goal of improving clinical outcomes in glioblastoma. 


\section{REFERENCES}

1. Ostrom, Q.T., et al., American Brain Tumor Association Adolescent and Young Adult Primary Brain and Central Nervous System Tumors Diagnosed in the United States in 2008-2012. Neuro Oncol, 2016. 18 Suppl 1: p. i1-i50.

2. Cha, S., Update on brain tumor imaging: from anatomy to physiology. AJNR Am J Neuroradiol, 2006. 27(3): p. 475-87

3. Young, G.S., Advanced MRI of adult brain tumors. Neurol Clin, 2007. 25(4): p. 947-73, viii.

4. Price, S.J. and J.H. Gillard, Imaging biomarkers of brain tumour margin and tumour invasion. $\mathrm{Br} \mathrm{J}$ Radiol, 2011. 84 Spec No 2: p. S159-67.

5. Brown, T.J., et al., Association of the Extent of Resection With Survival in Glioblastoma: A Systematic Review and Meta-analysis. JAMA Oncol, 2016. 2(11): p. 1460-1469.

6. Albert, F.K., et al., Early postoperative magnetic resonance imaging after resection of malignant glioma: objective evaluation of residual tumor and its influence on regrowth and prognosis. Neurosurgery, 1994. 34(1): p. 45-60; discussion 60-1.

7. Lacroix, M., et al., A multivariate analysis of 416 patients with glioblastoma multiforme: prognosis, extent of resection, and survival. J Neurosurg, 2001. 95(2): p. 190-8.

8. Stummer, W., et al., Extent of resection and survival in glioblastoma multiforme: identification of and adjustment for bias. Neurosurgery 2008. 62(3): p. 564-76; discussion 564-76.

9. Sanai, N. and M.S. Berger, Operative techniques for gliomas and the value of extent of resection Neurotherapeutics, 2009. 6(3): p. 478-86.

10. Wangaryattawanich, P., et al., Multicenter imaging outcomes study of The Cancer Genome Atlas glioblastoma patient cohort: imaging predictors of overall and progression-free survival. Neuro Oncol, 2015. 17(11): p. 1525-37.

11. Archive, T.C.I. VASARI Research Project. 3/25/2015 5/3/2017]; Available from: https:// wiki.cancerimagingarchive.net/display/Public/ VASARI+Research+Project.

12. Perez-Beteta, J., et al., Glioblastoma: does the pre-treatment geometry matter? A postcontrast T1 MRI-based study. Eur Radiol, 2017. 27(3): p. 1096-1104.

13. Czarnek, N., et al., Algorithmic three-dimensional analysis of tumor shape in MRI improves prognosis of survival in glioblastoma: a multi-institutional study. J Neurooncol, 2017. 132(1): p. 55-62.

14. Sanai, N., Z. Mirzadeh, and M.S. Berger, Functional outcome after language mapping for glioma resection. N Engl J Med, 2008. 358(1): p. 18-27.

15. Abdullah, K.G., et al., Use of diffusion tensor imaging in glioma resection. Neurosurg Focus, 2013. 34(4): p. E1.

16. Lee, S.K., Diffusion tensor and perfusion imaging of brain tumors in high-field MR imaging. Neuroimaging Clin N Am, 2012. 22(2): p. 123-34, ix.

17. Kim, R., et al., Prognosis prediction of nonenhancing T2 high signal intensity lesions in glioblastoma patients after standard treatment: application of dynamic contrast-enhanced MR imaging. Eur Radiol, 2017. 27(3): p. 1176-1185.
18. Chakravorty, A., T. Steel, and J. Chaganti, Accuracy of percentage of signal intensity recovery and relative cerebral blood volume derived from dynamic susceptibility-weighted, contrast-enhanced MRI in the preoperative diagnosis of cerebral tumours. Neuroradiol J, 2015. 28(6): p. $574-83$

19. Bakas, S., et al., In vivo detection of EGFRvIII in glioblastoma via perfusion magnetic resonance imaging signature consistent with deep peritumoral infiltration: the $\varphi$ index. Clinical Cancer Research, 2017, epub ahead of print.

20. Macyszyn, L., et al., Imaging patterns predict patient survival and molecular subtype in glioblastoma via machine learning techniques. Neuro Oncol, 2016. 18(3): p. 417-425.

21. Stupp, R., et al., Effects of radiotherapy with concomitant and adjuvant temozolomide versus radiotherapy alone on survival in glioblastoma in a randomised phase III study: 5-year analysis of the EORTC-NCIC trial. Lancet Oncol, 2009. 10(5): p. $459-66$.

22. Gilbert, M.R., et al., Dose-dense temozolomide for newly diagnosed glioblastoma: a randomized phase III clinical trial. J Clin Oncol, 2013. 31(32): p. 4085-91.

23. Stupp, R., et al., Radiotherapy plus concomitant and adjuvant temozolomide for glioblastoma. $N$ Engl J Med, 2005. 352(10): p. 987-96.

24. Wee, C.W., et al., Evaluation of variability in target volume delineation for newly diagnosed glioblastoma: a multi-institutional study from the Korean Radiation Oncology Group. Radiat Oncol, 2015. 10: p. 137.

25. Bagri, P.K., et al., Addition of magnetic resonance imaging to computed tomography-based threedimensional conformal radiotherapy planning for postoperative treatment of astrocytomas: Changes in tumor volume and isocenter shift South Asian J Cancer, 2015. 4(1): p. 18-20.

26. Harat, M., B. Malkowski, and R. Makarewicz, Pre-irradiation tumour volumes defined by MRI and dual time-point FET-PET for the prediction of glioblastoma multiforme recurrence: A prospective study. Radiother Oncol, 2016. 120(2): p. 241-7.

27. Elson, A., et al., Evaluation of pre-radiotherapy apparent diffusion coefficient (ADC): patterns of recurrence and survival outcomes analysis in patients treated for glioblastoma multiforme. $J$ Neurooncol, 2015. 123(1): p. 179-88.

28. Jeong, D., et al., Mean apparent diffusion coefficient values in defining radiotherapy planning target volumes in glioblastoma. Quant Imaging Med Surg, 2015. 5(6): p. 835-45

29. Qu, J., et al., Residual low ADC and high FA at the resection margin correlate with poor chemoradiation response and overall survival in highgrade glioma patients. Eur J Radiol, 2016. 85(3): p. 657-64.

30. Guo, L., et al., Diffusion and perfusion weighted magnetic resonance imaging for tumor volume definition in radiotherapy of brain tumors. Radiat Oncol, 2016. 11(1): p. 123.

31. Akbari, H., et al., Imaging Surrogates of Infiltration Obtained Via Multiparametric Imaging Pattern Analysis Predict Subsequent Location of Recurrence of Glioblastoma. Neurosurgery, 2016. 78(4): p. $572-80$
32. Deviers, A., et al., Evaluation of the lactate-to$\mathrm{N}$-acetyl-aspartate ratio defined with magnetic resonance spectroscopic imaging before radiation therapy as a new predictive marker of the site of relapse in patients with glioblastoma multiforme. Int J Radiat Oncol Biol Phys, 2014. 90(2): p. 385-93.

33. Cordova, J.S., et al., Whole-brain spectroscopic MRI biomarkers identify infiltrating margins in glioblastoma patients. Neuro Oncol, 2016. 18(8): p. 1180-9.

34. Macdonald, D.R., et al., Response criteria for phase II studies of supratentorial malignant glioma. J Clin Oncol, 1990. 8(7): p. 1277-80.

35. Grossman, R., et al., Dynamics of FLAIR Volume Changes in Glioblastoma and Prediction of Survival. Ann Surg Oncol, 2017. 24(3): p. 794-800.

36. Brandes, A.A., et al., MGMT promoter methylation status can predict the incidence and outcome of pseudoprogression after concomitant radiochemotherapy in newly diagnosed glioblastoma patients. J Clin Oncol, 2008. 26(13): p. 2192-7.

37. Wen, P.Y., et al., Updated response assessment criteria for high-grade gliomas: response assessment in neuro-oncology working group. J Clin Oncol, 2010. 28(11): p. 1963-72.

38. Chinot, O.L., et al., Response assessment criteria for glioblastoma: practical adaptation and implementation in clinical trials of antiangiogenic therapy. Curr Neurol Neurosci Rep, 2013. 13(5): p. 347.

39. Kreisl, T.N., et al., Phase II trial of single-agent bevacizumab followed by bevacizumab plus irinotecan at tumor progression in recurrent glioblastoma. J Clin Oncol, 2009. 27(5): p. 740-5.

40. Pope, W.B., et al., MRI in patients with highgrade gliomas treated with bevacizumab and chemotherapy. Neurology, 2006. 66(8): p. 1258-60.

41. Ananthnarayan, S., et al., Time course of imaging changes of GBM during extended bevacizumab treatment. J Neurooncol, 2008. 88(3): p. 339-47.

42. Okada, H., et al., Immunotherapy response assessment in neuro-oncology: a report of the RANO working group. Lancet Oncol, 2015. 16(15): p. e534-42.

43. Bennett, I.E., et al., Early perfusion MRI predicts survival outcome in patients with recurrent glioblastoma treated with bevacizumab and carboplatin. J Neurooncol, 2017. 131(2): p. 321-329.

44. O'Neill, A.F., et al., Demonstration of DCE-MRI as an early pharmacodynamic biomarker of response to VEGF Trap in glioblastoma. J Neuro Oncol, 2016. 130(3): p. 495-503.

45. Khalifa, J., et al., Identification of a candidate biomarker from perfusion MRI to anticipate glioblastoma progression after chemoradiation. Eur Radiol, 2016. 26(11): p. 4194-4203.

46. Kickingereder, P., et al., Large-scale Radiomic Profiling of Recurrent Glioblastoma Identifies an Imaging Predictor for Stratifying Anti-Angiogenic Treatment Response. Clin Cancer Res, 2016. 22(23): p. 5765-5771 